\title{
The Radiographic Union Score for Hip (RUSH) Identifies Radiographic Nonunion of Femoral Neck Fractures
}

\author{
Tym Frank MD, Georg Osterhoff MD, Sheila Sprague PhD, Alisha Garibaldi MSc, \\ Mohit Bhandari MD, PhD, Gerard P. Slobogean MD, MPH, on behalf of the FAITH Investigators
}

Published online: 4 January 2016

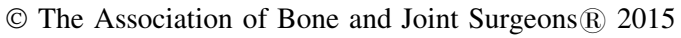

\begin{abstract}
Background The Radiographic Union Score for Hip (RUSH) is a previously validated outcome instrument designed to improve intra- and interobserver reliability when describing the radiographic healing of femoral neck fractures. The ability to identify fractures that have not healed is important for defining nonunion in clinical trials and predicting patients who will likely require additional surgery to promote fracture healing. We sought to investigate the utility of the RUSH score to define femoral neck fracture nonunion.

Questions/purposes (1) What RUSH score threshold yields at least $98 \%$ specificity to diagnose nonunion at 6 months postinjury? (2) Using the threshold identified, are patients below this threshold at greater risk of reoperation for nonunion and for other indications?

Methods A representative sample of 250 out of a cohort of 725 patients with adequate 6-month hip radiographs was
\end{abstract}

The FAITH trial was funded by research grants from the Canadian Institutes of Health Research (CIHR) (MB); National Institutes of Health (NIH) (PI: Marc Swiontkowski); Stichting NutsOhra (PI: Martin J. Heetveld), The Netherlands Organisation for Health Research and Development (PI: Esther M. M. Van Lieshout); and Physicians' Services Incorporated (PI: MB). One of the authors (MB) was also funded, in part, by a Canada Research Chair in Musculoskeletal Trauma, which is unrelated to the present study (McMaster University, Hamilton, Ontario, Canada). The FAITH study was supported by the National Institute of Arthritis and Musculoskeletal and Skin Diseases of the National Institutes of Health under Award Number R01AR055267. The County Durham \& Tees Valley Comprehensive Local Research Network, which operates as part of the National Institute for Health Research Comprehensive Clinical Research Network in England, also provided support to the FAITH study.

The content is solely the responsibility of the authors and does not necessarily represent the official views of the National Institutes of Health. analyzed from a multinational elderly hip fracture trial (FAITH). All patients had a femoral neck fracture and were treated with either multiple cancellous screws or a sliding hip screw. Two reviewers independently determined the RUSH score based on the 6-month postinjury radiographs and interrater reliability was assessed with the interclass correlation coefficient (ICC). There was substantial reliability between the reviewers assigning the RUSH scores (ICC, $0.81 ; 95 \%$ confidence interval [CI], 0.76-0.85). The RUSH score is a checklist-based system that quantifies four measures of healing: cortical bridging, cortical fracture disappearance, trabecular consolidation, and trabecular fracture disappearance.. Fracture healing was determined by two independent methods: (1) concurrently by the treating surgeon using both clinical and radiographic assessments as per routine clinical care; and (2) retrospectively by a Central Adjudication Committee using complete obliteration of the fracture line on radiographs alone. Receiver operating characteristic tables were used to

All ICMJE Conflict of Interest Forms for authors and Clinical Orthopaedics and Related Research ${ }^{\mathbb{R}}$ editors and board members are on file with the publication and can be viewed on request.

Each author certifies that his or her institution approved the human protocol for this investigation, that all investigations were conducted in conformity with ethical principles of research, and that informed consent for participation in the study was obtained.

This study was coordinated at the Centre for Evidence Based Orthopaedics, McMaster University, Hamilton, Ontario, Canada. See Appendix for a list of the FAITH Investigators.

T. Frank, G. Osterhoff

Department of Orthopaedics, University of British Columbia, Vancouver, BC, Canada

S. Sprague, A. Garibaldi, M. Bhandari

Department of Clinical Epidemiology and Biostatistics,

McMaster University, Hamilton, ON, Canada 
define a RUSH threshold score that was $>98 \%$ specific for fracture nonunion.

Results A threshold score of $<18$ was associated with a $100 \%$ specificity $(95 \%$ CI, $97 \%-100 \%)$ and a positive predictive value of $100 \%$ (95\% CI, $73 \%-100 \%$ ) for radiographic nonunion. In contrast, using the fracture healing assessments of the treating surgeons failed to identify a useful discriminatory nonunion threshold and the highest positive predictive value was $43 \%$. With respect to complications, patients with RUSH scores below 18 had greater risk of undergoing reoperation for nonunion (reoperation when < 18: six of 13 [46\%]; reoperation when $\geq 18$ : 11 of 237 [54\%]; relative risk [RR], 9.9 [95\% CI, 4.4-22.7]; $p<$ 0.001 ) and for all indications (reoperation when $<18$ : eight of 13 [62\%]; reoperation when $\geq 18: 54$ of 237 [38\%]; RR, 2.7 [95\% CI, 1.7-4.4]; $\mathrm{p}=0.004$ ).

Conclusions The 6-month RUSH score is a reliable method for assessing radiographic healing. Our results highlight the discordance between radiographic determinations and clinician assessments of fracture healing and stress the need for clinical data to be incorporated in research studies evaluating fracture healing.

Level of Evidence Level III, diagnostic study.

\section{Introduction}

Clinicians, researchers, and federal regulators have often failed to agree on relevant and reproducible measures of fracture healing [8]. Although most surgeons believe fracture healing should be determined using clinical and radiographic information, substantial variability in the methods for assessing healing is observed in practice and the research environment $[3,7,11,15,16]$. This is extremely problematic when trying to establish the validity of a study's results or make comparative evaluations between different studies. As a result of the high incidence of elderly hip fractures and its associated morbidity [9, 12, 14], numerous clinical trials are aimed at interventions to improve femoral neck fracture healing outcomes; however, the assessment of femoral neck fracture healing remains highly subjective and causes disagreements among specialists [6].

S. Sprague, A. Garibaldi, M. Bhandari

The Division of Orthopaedic Surgery, Department of Surgery, McMaster University, Hamilton, ON, Canada

G. P. Slobogean $(\square)$

Department of Orthopaedics, University of Maryland School of Medicine, 6th Floor, Suite 300, 110 S Paca Street, Baltimore, MD 21201, USA

e-mail: gslobogean@umoa.umm.edu
A variety of descriptions for fracture nonunion have been proposed in recent clinical studies, including a past US Department of Health and Human Service's definition of nonunion, "when fracture healing has ceased for 3 or more months" [10]. Although many authors argue that a temporal definition for fracture healing is too arbitrary and flawed [4], describing a minimum amount of cortical and trabecular bridging to distinguish between healed and ununited fractures is equally as subjective. Many healed fractures may not achieve complete trabecular consolidation and cortical bridging despite the patient's return to full activities. Clinically, surgeons use radiographic and patient assessments to determine if a fracture has united.

The Radiographic Union Score for Hip (RUSH) is a previously validated tool that improves fracture healing agreement between radiologists and orthopaedic surgeons by using a checklist-based scoring approach $[1,2,5]$. Using this tool to grade the healing of femoral neck fractures leads to better agreement with respect to radiographic healing and improved intra- and interobserver reliability [5]. We sought to determine if this systematic and standardized scoring approach could be used to determine fracture healing and predict clinically important outcomes. Therefore, the primary purpose of this study was to find a RUSH score that is highly specific for femoral neck nonunion at 6-months and, secondarily, to determine if this score would be associated with an increased risk for reoperation.

We therefore asked: (1) What RUSH score threshold yields at least $98 \%$ specificity to diagnose nonunion at 6 months postinjury? (2) Using the threshold identified, are patients below this threshold at greater risk of reoperation for nonunion repair and for other indications?

\section{Materials and Methods}

We used a convenience sample from the ongoing Fixation Using Alternative Implants for the Treatment of Femoral Neck Fractures (FAITH) trial [8]. FAITH is a prospective randomized controlled trial comparing multiple cancellous screws versus the sliding hip screw for the treatment of elderly femoral neck fracture patients (ClinicalTrials.gov Identifier, NCT00761813). This trial is coordinated by the Centre for Evidenced-Based Orthopaedics (McMaster University) and has been approved by the Hamilton Integrated Research Ethics Board (\#06-402). All radiographs and measures of fracture healing were obtained prospectively as part of the trial's protocol. Using the study radiographs, two authors (TF, GO) independently assigned a RUSH score to the 6-month postinjury radiographs of all included patients. The reviewers were blinded to study outcome events and all analyses were performed using fracture healing data obtained from the trial. 
To be eligible for the current study, patients were required to have adequate AP and lateral hip radiographs at 6 months (followup window, 5-7 months) and to have all study outcome data reviewed by the independent Central Adjudication Committee (CAC). Adequate radiographs were those that had sufficient penetrance and image quality such that the components of the RUSH score could be reliably assessed. The 6-month followup visit was selected as the time point of interest for our study because it was felt to be the first clinical visit that unhealed fractures could be declared a nonunion and, similarly, any reoperations that occurred before 6 months would likely have been for indications other than nonunion. A representative sample of 250 trial participants were included in our analysis out of a possible 1112 enrolled in the FAITH trial. Four hundred seventy-five patients were excluded for inadequate or missing 6-month followup radiographs. The remaining 387 patients had not completed their 2-year study followup or their clinical data had not yet been analyzed by the CAC.

Our primary clinical outcome was nonunion at 6-months, as defined by an ununited femoral neck fracture at the 6month assessment. We acknowledged that defining a femoral neck nonunion as any ununited fracture at 6 months is a simplistic determination but was appropriate for the context of the study design. Nonunion events were obtained from two sources: 1) the treating surgeon's assessment at the point of care; and 2) the CAC's assessment of fracture healing based on radiographs only. The assessment of fracture healing by the local treating surgeon was used as the primary outcome defining nonunion at 6 months. We opted to use the local assessment of fracture healing because the treating surgeon had the benefit of using the radiographic and clinical examination to make his or her determination.

The CAC consisted of five experienced orthopaedic trauma surgeons (GPS, GDR, SL, KJ, RH) trained in outcome adjudication. The committee retrospectively viewed all available radiographs and case report forms describing study outcome events such as reoperation. The CAC made a consensus determination of either "healed" or "not healed" at each study time point based solely on radiographic parameters. A fracture was considered healed when there was complete obliteration of the fracture line on the radiograph. This was intentionally a conservative assessment because the CAC was unable to evaluate the patients clinically, unlike the local surgeon. Details surrounding the indication for reoperation were reviewed by the CAC to ensure this met the criteria for a study event, namely the reoperation, was unplanned and related to the femoral neck fracture.

Although we recognized that using the treating surgeon's assessment may introduce local bias or increased intersurgeon variability in the determination of fracture healing, the additional use of the CAC data would provide a secondary assessment to further validate the findings of our study. The repeat analysis using the CAC's assessment of healing revealed similar findings to surgeon assessment (Figs. 1, 2). As expected, the CAC's more stringent definition of radiographic healing resulted in a larger proportion of fractures being classified as a nonunion at 6 months (CAC: $n=122$ of 250 [49\%], treating surgeons: $n=53$ of 250 [21\%]; $p<0.001$ ); however, the mean RUSH score of the nonunion group was similar to the result using the local surgeon assessment (CAC: $22.6 \pm 3.7$, treating surgeons: $22.1 \pm 4.0 ; \mathrm{p}=0.15)$.

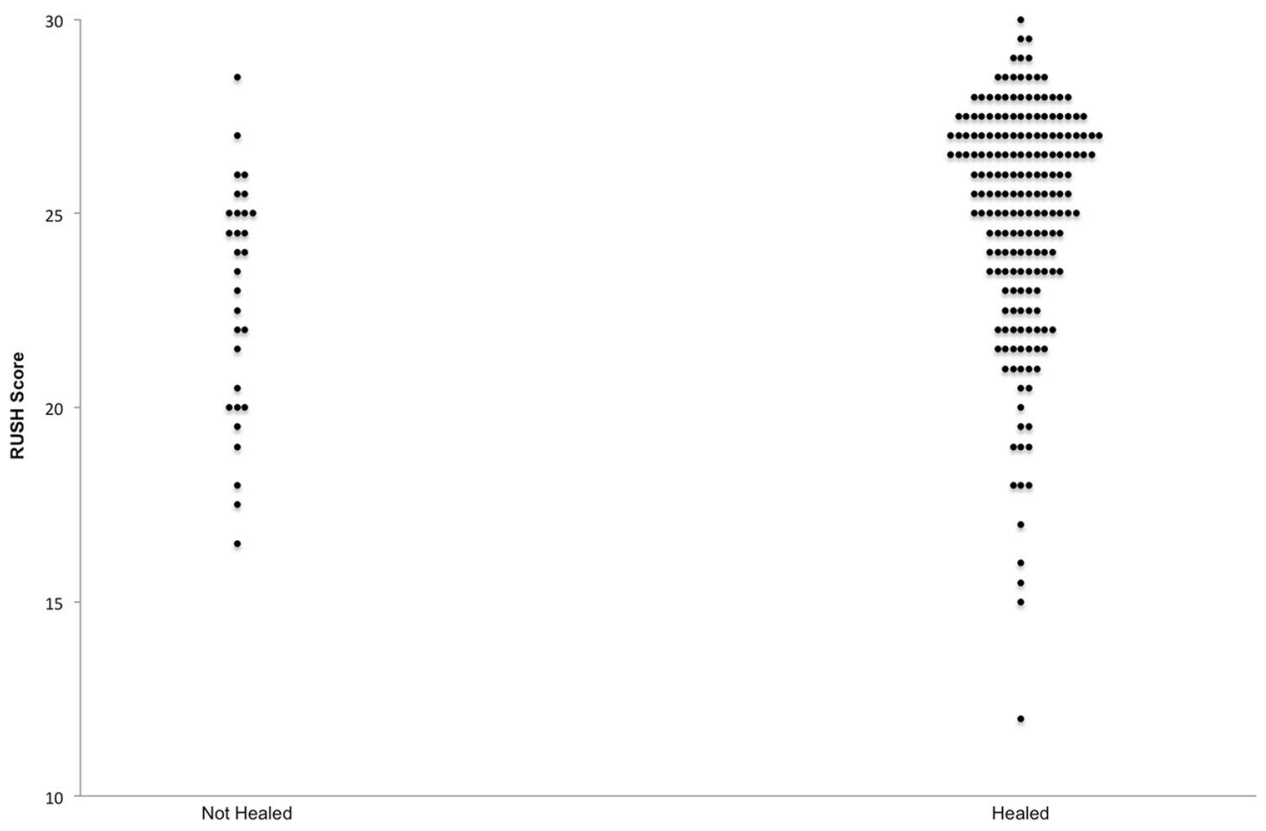

Fig. 1 The RUSH scores based on the treating physicians' assessment are stratified by fracture healing in the scatterplot. 


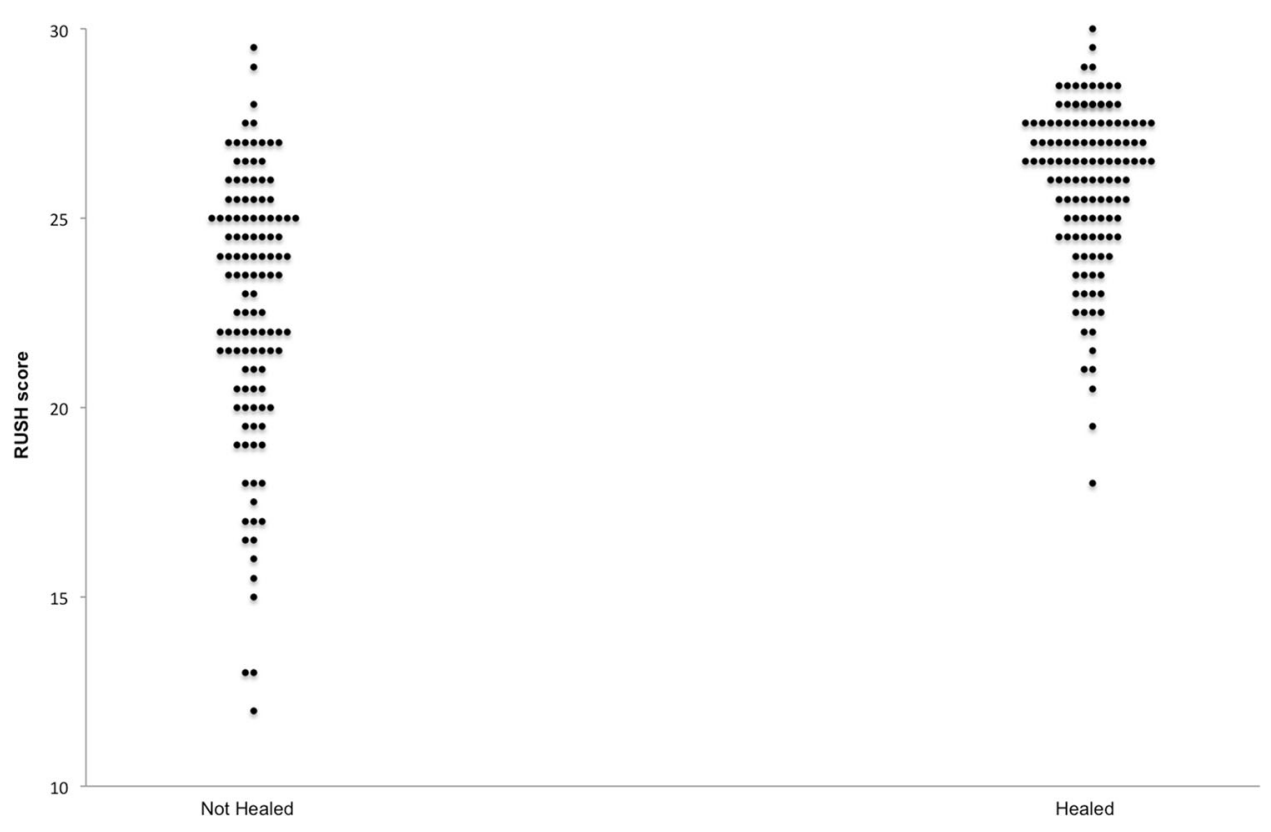

Fig. 2 The RUSH scores based on the CAC's radiographic assessment are stratified by fracture healing in the scatterplot.

Two investigators (TF, GO) reviewed the 6-month radiographs of all included patients and independently assigned a RUSH score for each patient. The reviewers were provided with the original publications describing the methods for assigning a RUSH and a brief tutorial to promote consistency. The RUSH quantifies four measures of healing: cortical bridging, cortical fracture disappearance, trabecular consolidation, and trabecular fracture disappearance [3]. Cortical healing is assessed in four anatomic femoral neck regions (anterior, posterior, medial, lateral) and trabecular healing is measured with two assessments (fracture line disappearance and consolidation of matrix). Each of the 10 assessed dimensions of radiographic femoral neck healing are scored 1 to 3 , leading to a minimum score of 10 (no signs of healing) and a maximum score of 30 (perfect healing) (Fig. 3). The average RUSH score between the two reviewers' assessments was used as the final RUSH score for all analyses. Interobserver agreement of RUSH scores was assessed by the interclass coefficient (ICC 2,k) to ensure adequate agreement. An ICC of $>0.8$ was used to define nearly perfect agreement, as suggested by Landis and Koch [13]. There was substantial reliability between the reviewers assigning the RUSH scores (ICC, $0.81 ; 95 \%$ confidence interval [CI], 0.76-0.85). The mean 6-month RUSH score for the entire cohort was $24.3 \pm 3.4$.
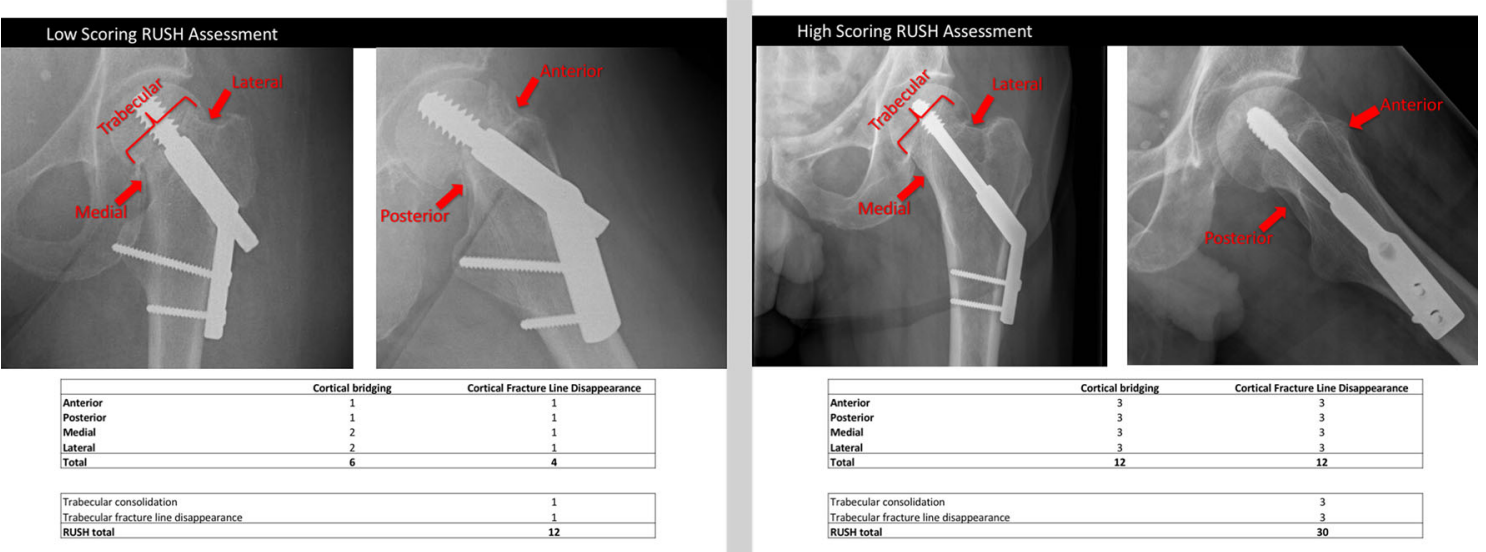

Fig. 3 Radiographs taken at 6 months postinjury serve as examples for a low-scoring RUSH assessment (RUSH: 12) and a high-scoring RUSH assessment (RUSH: 30). 


\section{Statistical Analysis}

All data were compiled in an Excel database (Microsoft Corp, Redmond, WA, USA) and exported to JMP 9.0 (SAS Institute Inc, Cary, NC, USA) for statistical analysis. Unless otherwise denoted, data were summarized with its mean and SD or as proportions with $95 \%$ CIs. A Student's t-test was used to test for difference in RUSH scores between subgroups. The level of significance was defined as $\mathrm{p}<0.05$. The primary analysis was performed using the treating surgeons' assessment of fracture healing at 6 months and the average RUSH score for the corresponding 6-month hip radiographs. Secondary associations with the 6-month RUSH score were examined using the CAC's assessment of 6-month fracture healing as well as reoperations between 6 months and 2 years postinjury for nonunion and all-cause reoperation indications such as implant removal.

Receiver operating characteristic tables were computed to examine the specificity of threshold RUSH values that would correctly classify a nonunion. Because the study's primary objective was to identify a threshold RUSH score that would be specific for nonunion, we sought to maximize the specificity of the threshold RUSH value. This would ensure all radiographs with a RUSH score below the defined threshold would be classified as nonunions. A priori, we decided that a threshold with $>98 \%$ specificity would represent a clinically acceptable margin of error and would meet our study objective. Finally, the positive predictive value $(\mathrm{PPV}=$ true-positives/true-positives + falsepositives) was calculated to assess the discriminatory value of our threshold for defining nonunion.

\section{Results}

The mean age of study participants was $71 \pm 12$ years and two-thirds of the fractures were Garden I or II fracture patterns (Table 1). A total of 121 patients received a sliding hip screw and 129 received multiple cancellous screws.

A RUSH score of $<18$ corresponds to $100 \%$ specificity and a PPV of $100 \%$ with 13 cases meeting criteria. Increasing specificity is achieved by decreasing the RUSH threshold score for both the treating surgeon and the CAC assessments of healing (Table 2). According to the treating surgeons' assessments, 53 patients $(21 \%)$ had a femoral neck nonunion at 6 months. The mean RUSH score was 2.8 points higher (95\% CI, 1.7-4.0) among healed fractures compared to ununited fractures $(22.1 \pm 4.0$ versus $24.9 \pm$ 3.0, respectively) (Fig. 1). Based on the treating surgeons' assessment of fracture healing, a 6-month RUSH score of less than 16 points defines a nonunion; however, this
Table 1. Participant demographics $(n=250)$

\begin{tabular}{ll}
\hline Variable & Number $(\%)$ \\
\hline Mean age \pm SD (years) & $71 \pm 12$ \\
Sex & \\
Female & $153(61)$ \\
Male & $97(39)$ \\
Fracture pattern* & \\
Type I & $133(53)$ \\
Type II & $36(14)$ \\
Type III & $57(23)$ \\
Type IV & $21(8)$ \\
Unable to assess & $3(1)$ \\
Mechanism of injury & \\
Fall & $237(94)$ \\
Trauma & $6(2)$ \\
Spontaneous & $5(2)$ \\
Unknown & $2(1)$ \\
Reoperation & \\
All causes & $62(25)$ \\
Nonunion & $17(7)$ \\
\hline
\end{tabular}

* Garden classification is used to describe the fracture pattern.

diagnostic threshold has a poor PPV (43\%) and only six cases in the entire series met this definition.

Patients with a RUSH score of $<18$ were 10 times more likely to undergo a nonunion reoperation than individuals with higher scores (relative risk [RR], 9.9; 95\% CI, 4.422.7). Similarly, the 18-point threshold was also predictive for reoperation for all indication (RR, 2.7; 95\% CI, 1.74.4).

The repeat analysis using the CAC's assessment of healing revealed similar trends (Fig. 2). As expected, the CAC's more stringent definition of radiographic healing resulted in a larger proportion of fractures being classified as nonunion at 6 months $(n=122[49 \%])$; however, the mean RUSH score was approximately 3 points higher among healed fractures versus nonunions $(\mathrm{p}<0.001)$.

\section{Discussion}

The assessment of femoral neck fracture healing remains highly subjective and causes disagreements among specialists. The RUSH score is an instrument designed to describe radiographic healing of femoral neck fractures. The impetus for this study was the desire to expand the utility of the RUSH score to standardize a reproducible definition of fracture nonunion and to improve the ability of describing the continuum of radiographic healing in this metaphyseal region. By describing a method to assess cortical and trabecular healing, previous studies have 
Table 2. Threshold RUSH score for defining nonunion

\begin{tabular}{|c|c|c|c|c|c|c|c|c|}
\hline \multirow[t]{2}{*}{ RUSH score } & \multicolumn{4}{|c|}{ Surgeon assessment } & \multicolumn{4}{|l|}{ CAC assessment } \\
\hline & Specificity (\%) & $95 \%$ CI (\%) & PPV (\%) & $95 \%$ CI (\%) & Specificity (\%) & $95 \%$ CI (\%) & PPV (\%) & $95 \%$ CI (\%) \\
\hline 20 & 93 & $88-96$ & 53 & $34-72$ & 98 & $94-100$ & 93 & $78-99$ \\
\hline 19 & 94 & $90-97$ & 48 & $26-70$ & 99 & $96-100$ & 95 & $76-100$ \\
\hline 18 & 96 & $92-98$ & 53 & $28-77$ & 99 & $96-100$ & 94 & $71-100$ \\
\hline 17 & 97 & 94-99 & 58 & $28-85$ & 100 & $97-100$ & 100 & $73-100$ \\
\hline 16 & 98 & 95-99 & 43 & $10-82$ & 100 & $97-100$ & 100 & 79-100 \\
\hline
\end{tabular}

RUSH = Radiographic Union Score for Hip; $\mathrm{CAC}=$ Central Adjudication Committee; $\mathrm{CI}=$ confidence interval; $\mathrm{PPV}=$ positive predictive value .

demonstrated excellent inter- and intrarater reliability of the instrument; however, the RUSH score has not been used to define fracture healing. In the current study, we aimed to determine a RUSH threshold score that would correctly classify femoral neck fractures that have not healed at 6 months.

There is no gold standard for the assessment of femoral neck fracture healing and this remains problematic. Although radiographic healing has traditionally been an important outcome of interest, the current results demonstrate a large discordance between clinical practice and determining healing outcomes solely by radiographs. As a result, we believe this observation continues to stress the necessity of orthopaedic research to be based on clinical data and patient-reported outcomes. Furthermore, it must also be mentioned that both RUSH score assessors knew that all radiographs were taken at 6 months postinjury. Although this is not a limitation of the study's internal validity, it is important that readers recognize that the findings likely do not apply to radiographs earlier than 6 months postinjury. We also acknowledged that defining a femoral neck nonunion as any ununited fracture at 6 months is a simplistic determination but was appropriate for the context of the study design. Additionally, although we captured a representative sample of 250 out of a possible 725 patients, the rather large number of exclusions was attributed to lack of sufficient radiograph quality for accurate RUSH score assessment and this highlights a possible limitation to using the RUSH. Finally, although we were only able to analyze the radiographs of 250 patients, we do not believe there is any reason to suspect this sample of the study population would lead to biases in the RUSH scores assigned or the external validity of our results.

Our results suggest that a RUSH score of less than 18 has a $100 \%$ PPV for defining radiographic nonunion at 6 months postinjury; however, when one defines fracture healing using the treating surgeons' combined clinical and radiographic assessment, there is no RUSH threshold that is useful for classifying femoral neck fracture no-unions.
The discrepancy between the local surgeons' assessment and the radiograph-only assessment (CAC) highlights the heavy influence clinical evaluation imparts on a surgeon's determination of fracture healing. The use of the CAC definition of healing was intentionally conservative; however, the assessment of the PPV is dependent on the prevalence of nonunion in the sample. Because the nonunion rate changes with certain populations, this will inevitably have an effect on the PPV of the 18-point RUSH threshold. The use of multicenter data for our study population increases the confidence that our observed prevalence of nonunion is generalizable to most populations; however, one would expect the prevalence, and therefore the PPV, to change depending on differences in the proportion of displaced fractures.

The RUSH score threshold of 18 also demonstrates utility in predicting reoperations for nonunions as well as reoperations for any other indication. RUSH scores below this threshold were 10 times more likely to undergo a nonunion reoperation than individuals with higher scores, and as expected, we found lower 6-month RUSH scores among patients who experienced a reoperation after their 6-month visit, both for indications of nonunion $(19.6 \pm 4.9$ versus 24.7 $\pm 3.0)$ and all-cause indications (22.9 \pm 4.2 versus $24.8 \pm$ $3.0, \mathrm{p}=0.002$ ). This finding suggests again that the radiographic parameters of a femoral neck fracture at 6 months measured by the RUSH score are reliable for assessing radiographic healing but the discrepancy when using treating surgeon definitions of nonunion underlines the finding that this may not necessarily tell the entire clinical picture.

The 6-month RUSH score is a reliable method for assessing radiographic healing. Our results highlight the discordance between radiographic determinations and clinician assessments of fracture healing and stress the need for clinical data to be incorporated in research studies evaluating fracture healing.

Acknowledgments We thank Dr Gregory Della Rocca, Miss Susan Liew, Dr Kyle Jeray, and Dr Robert Haverlag for independently reviewing all FAITH study radiographs and adjudicating study outcome events. 


\section{Appendix: FAITH Investigators}

Writing Committee: Tym Frank, Georg Osterhoff, Sheila Sprague, Alisha Garibaldi, Mohit Bhandari, Gerard Slobogean.

Steering Committee: Mohit Bhandari (Chair), Marc Swiontkowski, P. J. Devereaux, Gordon Guyatt, Martin J. Heetveld, Kyle Jeray, Susan Liew, Emil H. Schemitsch, Lehana Thabane, Stephen Walter.

Global Methods Centre: Mohit Bhandari (Principal Investigator); Sheila Sprague (Research Methodologist); Paula McKay (Manager); Taryn Scott, Alisha Garibaldi, Helena Viveiros, Marilyn Swinton (Research Coordination); Mark Gichuru and Sofia Bzovsky (Adjudication Coordination); Diane Heels-Ansdell, Qi Zhou (Statistical Analysis); Lisa Buckingham, Aravin Duraikannan (Data Management); Deborah Maddock, Nicole Simunovic (Grants Management) (McMaster University) (McMaster University).

US Methods Centre: Marc Swiontkowski (Principal Investigator); Julie Agel (Research Coordination) (University of Minnesota).

Netherlands Method Centre: Martin J. Heetveld (Principal Investigator); Esther M.M. Van Lieshout (Research Coordination); Stephanie M. Zielinski (Trial Coordination) (Erasmus MC, University Medical Center Rotterdam).

UK Method Centre: Amar Rangan (Principal Investigator), Birgit C. Hanusch, Lucksy Kottam, Rachel Clarkson (Research Coordination) (The James Cook University Hospital).

Central Adjudication Committee: Gregory J Della Rocca (Chair), Susan Liew, Kyle Jeray, Robert Haverlag, Gerard Slobogean.

Data Safety Monitoring Board: Jeffrey Katz (Chair), Brenda Gillespie, Gail A. Greendale, Pierre Guy, Curtis Hartman, Craig Rubin, James Waddell.

The following persons participated in the FAITH Study:

\section{Canada:}

Robert McCormack, Kelly Apostle, Dory Boyer, Farhad Moola, Bertrand Perey, Trevor Stone, Darius Viskontas, H. Michael Lemke, Mauri Zomar, Karyn Moon, Raely Moon, Amber Oatt (University of British Columbia/Fraser Health Authority); Richard E. Buckley, Paul Duffy, Robert Korley, Shannon Puloski, Kelly Johnston, James Powell, Kimberly Carcary (Foothills Medical Centre); David Sanders, Abdel Lawendy, Christina Tieszer (London Health Sciences Centre); David Stephen, Hans Kreder, Richard Jenkinson, Markku Nousiainen, Terry Axelrod, John Murnaghan, Diane Nam, Veronica Wadey, Albert Yee, Katrine Milner, Monica Kunz (Sunnybrook Health Sciences Centre); Emil H. Schemitsch, Henry Ahn, Jeremy A. Hall, Michael D. McKee, Daniel B. Whelan, Aaron Nauth, Milena R. Vicente, Lisa M. Wild, Ryan M. Khan, Jennifer
T. Hidy (St Michael's Hospital); Chad Coles, Ross Leighton, Michael Biddulph, David Johnston, Mark Glazebrook, David Alexander, Cathy Coady, Michael Dunbar, David Amirault, Michael Gross, William Oxner, Gerald Reardon, Glen Richardson, Andrew Trenholm, Ivan Wong, Kelly Trask, Shelley MacDonald, Gwen Dobbin (Queen Elizabeth II Health Sciences Centre); Ryan Bicknell, Jeff Yach, Davide Bardana, Gavin Wood, Mark Harrison, David Yen, Sue Lambert, Fiona Howells, Angela Ward (Human Mobility Research Centre, Queen's University and Kingston General Hospital); Paul Zalzal, Heather Brien, V. Naumetz, Brad Weening, Nicole Simunovic (Oakville Trafalgar Memorial Hospital); Eugene K. Wai, Steve Papp, Wade T. Gofton, Allen Liew, Stephen P. Kingwell, Garth Johnson, Joseph O'Neil, Darren M. Roffey, Vivian Borsella (Ottawa Hospital); Victoria Avram (Juravinski Hospital and Cancer Centre).

\section{United States:}

Todd M. Oliver, Vicki Jones (Boone Hospital CenterColumbia Orthopaedic Group); Clifford B. Jones, James R. Ringler, Terrence J. Endres, Samuel G. Agnew, Debra L. Sietsema, Samuel G. Agnew, Jane E. Walker (Orthopaedic Associates of Michigan); Kyle J. Jeray, J. Scott Broderick, David R. Goetz, Thomas B. Pace, Thomas M. Schaller, Scott E. Porter, Stephanie L. Tanner, Rebecca G. Snider, Lauren A. Nastoff, Shea A. Bielby (Greenville Hospital System); Julie A. Switzer, Peter A. Cole, Sarah A. Anderson, Paul M. Lafferty, Mengnai Li, Thuan V. Ly, Scott B. Marston, Amy L. Foley, Sandy Vang, David M. Wright (Regions HospitalUniversity of Minnesota); Andrew J. Marcantonio, Michael S. H. Kain, Richard Iorio, Lawrence M. Specht, John F. Tilzey, Margaret J. Lobo, John S. Garfi (Lahey Hospital \& Medical Center); Heather A. Vallier, Andrea Dolenc, Chalitha Robinson (MetroHealth Medical Center); Michael J. Prayson, Richard Laughlin, L. Joseph Rubino, Jedediah May, Geoffrey Ryan Rieser, Liz Dulaney-Cripe, Chris Gayton (Miami Valley Hospital); James Shaer, Tyson Schrickel, Barbara Hileman (St Elizabeth Youngstown Hospital); John T. Gorczyca, Jonathan M. Gross, Catherine A. Humphrey, Stephen Kates, Krista Noble, Allison W. McIntyre, Kaili Pecorella (University of Rochester Medical Center); Craig A. Davis, Stewart Weinerman, Peter Weingarten, Philip Stull, Stephen Lindenbaum, Michael Hewitt, John Schwappach, Janell K. Baker, Tori Rutherford, Heike Newman, Shane Lieberman, Erin Finn, Kristin Robbins, Meghan Hurley, Lindsey Lyle, Khalis Mitchell, Kieran Browner, Erica Whatley, Krystal Payton, Christina Reeves (Colorado Orthopedic Consultants); Lisa K. Cannada, David Karges, Leslie Hill (St Louis University Hospital); Samir Mehta, John Esterhai, Jaimo Ahn, Annamarie D. Horan, Kelly McGinnis, Christine A. Kaminski, Brynn N. Kowalski (University of Pennsylvania); Jonathan P. Keeve, Christopher G. Anderson, 
Michael D. McDonald, Jodi M. Hoffman (Northwest Orthopaedic Specialists); Ivan Tarkin, Peter Siska, Gary Gruen, Andrew Evans, Dana J. Farrell, James Irrgang, Arlene Luther (University of Pittsburgh Medical Center); William W. Cross III, Joseph R. Cass, Stephen A. Sems, Michael E. Torchia, Tyson Scrabeck (Mayo Clinic); Mark Jenkins, Jules Dumais, Amanda W. Romero (Texas Tech University Health Sciences Center-Lubbock); Carlos A. Sagebien, Mark S. Butler, James T. Monica, Patricia Seuffert (University Orthopaedic Associates, LLC); Joseph R. Hsu, James Ficke, Michael Charlton, Matthew Napierala, Mary Fan (US Army Institute of Surgical Research); Paul Tornetta III, Chadi Tannoury, Hope Carlisle, Heather Silva (Boston University Medical Center); Michael Archdeacon, Ryan Finnan, Toan Le, John Wyrick, Shelley Hess (UC Health/University of Cincinnati Medical Center); Michael L. Brennan, Robert Probe, Evelyn Kile, Kelli Mills, Lydia Clipper, Michelle Yu, Katie Erwin (Scott and White Memorial Hospital); Daniel Horwitz, Kent Strohecker, Teresa K. Swenson (Geisinger Medical Center); Andrew H. Schmidt, Jerald R. Westberg (Hennepin County Medical Center); Kamran Aurang, Gary Zohman, Brett Peterson, Roger B. Huff (Kaiser Permanente); Joseph Baele, Timothy Weber, Matt Edison (OrthoIndy); Jessica McBeth (Santa Clara Valley Medical Center); Karl Shively, Janos P. Ertl, Brian Mullis, J. Andrew Parr, Ripley Worman, Valda Frizzell, Molly M. Moore, Erin Tobias, Emily Thomas (Indiana University-Wishard Health Services); Charles J. DePaolo, Rachel Alosky, Leslie E. Shell, Lynne Hampton, Stephanie Shepard, Tracy Nanney, Claudine Cuento (Mission Hospital Research Institute); Robert V. Cantu, Eric R. Henderson, Linda S. Eickhoff (DartmouthHitchcock Medical Center); E. Mark Hammerberg, Philip Stahel, David Hak, Cyril Mauffrey, Douglas Gibula, Hannah Gissel, Corey Henderson (Denver Health and Hospital Authority); David P. Zamorano, Martin C. Tynan, Deeba Pourmand, Deanna Lawson (University of California Irvine Medical Center); Gregory J. Della Rocca, Brett D. Crist, Yvonne M. Murtha, Linda K. Anderson (University of Missouri Health Care); Colleen Linehan, Lindsey Pilling (Covenant Healthcare of Saginaw); Courtland G. Lewis, Stephanie Caminiti, Raymond J. Sullivan, Elizabeth Roper (Hartford Hospital); William Obremskey, Philip Kregor, Justin E. Richards, Kenya Stringfellow (Vanderbilt University Medical Center); Michael P. Dohm, Abby Zellar (Western Slope Study Group).

\section{The Netherlands:}

Michiel J. M. Segers, Jacco A. C. Zijl, Bart Verhoeven, Anke B. Smits, Jean Paul P. M. de Vries, Bram Fioole, Henk van der Hoeven, Evert B. M. Theunissen, Tammo S. de Vries Reilingh, Lonneke Govaert, Philippe Wittich, Maurits de Brauw, Jan Wille, Peter M. N. Y. M. Go, Ewan D. Ritchie, Ronald N. Wessel, Eric R. Hammacher (St
Antonius Ziekenhuis); Martin J. Heetveld, Gijs A. Visser, Heyn Stockmann, Rob Silvis, Jaap P. Snellen, Bram Rijbroek, Joris J. G. Scheepers, Erik G. J. Vermeulen, Michiel P. C. Siroen, Ronald Vuylsteke, Hans L. F. Brom, Herman Rijna (Kennemer Gasthuis); Piet A. R. de Rijcke, Cees L. Koppert, Steven E. Buijk, Richard P. R. Groenendijk, Imro Dawson, Geert W. M. Tetteroo, Milko M. M. Bruijninckx, Pascal G. Doornebosch, Eelco J. R. de Graaf (IJsselland Ziekenhuis); Maarten van der Elst, Carmen C. van der Pol, Martijne van 't Riet, Tom M. Karsten, Mark R. de Vries, Laurents P. S. Stassen, Niels W. L. Schep, G. Ben Schmidt, W. H. Hoffman (Reinier de Graaf Gasthuis); Rudolf W. Poolman, Maarten P. Simons, Frank H. W. M. van der Heijden, W. Jaap Willems, Frank R. A. J. de Meulemeester, Cor P. van der Hart, Kahn Turckan, Sebastiaan Festen, Frank de Nies, Robert Haverlag, Nico J. M. Out, Jan Bosma (Onze Lieve Vrouwe Gasthuis); Albert van Kampen, Jan Biert, Arie B. van Vugt, Michael J. R. Edwards, Taco J. Blokhuis, Jan Paul M. Frölke, Leo M. G. Geeraedts, Jean W. M. Gardeniers, Edward T. C. H. Tan, Lodewijk M. S. J. Poelhekke, Maarten C. de Waal Malefijt, Bart Schreurs (University Medical Center St Radboud); Gert R. Roukema, Hong A. Josaputra, Paul Keller, Peter D. de Rooij, Hans Kuiken, Han Boxma, Berry I. Cleffken, Ronald Liem (Maasstad Ziekenhuis); Steven J. Rhemrev, Coks H. R. Bosman, Alexander de Mol van Otterloo, Jochem Hoogendoorn, Alexander C. de Vries, Sven A. G. Meylaerts (Medisch Centrum Haaglanden); Michiel H. J. Verhofstad, Joost Meijer, Teun van Egmond, Frank H. W. M. van der Heijden, Igor van der Brand (St Elisabeth Ziekenhuis); Peter Patka, Martin G. Eversdijk, Rolf Peters, Dennis Den Hartog, Oscar J. F. Van Waes, Pim Oprel (Erasmus MC, University Medical Center Rotterdam); Harm M. van der Vis, Martin Campo, Ronald Verhagen, G. H. Robert Albers, Arthur W. Zurcher (Tergooi Ziekenhuizen); Rogier K. J. Simmermacher, Jeroen van Mulken, Karlijn van Wessem, Taco J. Blokhuis, Steven M. van Gaalen, Luke P. H. Leenen (University Medical Center Utrecht); Maarten W. G. A. Bronkhorst, Onno R. Guicherit (Bronovo Ziekenhuis); J. Carel Goslings, Robert Haverlag, Kees Jan Ponsen (Academic Medical Center).

\section{International:}

Mahesh Bhatia, Vinod Arora, Vivek Tyagi (RLB Hospital and Research Centre, India); Susan Liew, Harvinder Bedi, Ashley Carr, Hamish Curry, Andrew Chia, Steve Csongvay, Craig Donohue, Stephen Doig, Elton Edwards, Greg Etherington, Max Esser, Andrew Gong, Arvind Jain, Doug Li, Russell Miller, Ash Moaveni, Matthias Russ, Lu Ton, Otis Wang, Adam Dowrick, Zoe Murdoch, Claire Sage (The Alfred, Australia); Frede Frihagen, John Clarke-Jenssen, Geir Hjorthaug, Torben Ianssen, Asgeir Amundsen, Jan Egil Brattgjerd, Tor Borch, 
Berthe Bøe, Bernhard Flatøy, Sondre Hasselund, Knut Jørgen Haug, Kim Hemlock, Tor Magne Hoseth, Geir Jomaas, Thomas Kibsgård, Tarjei Lona, Gilbert Moatshe, Oliver Müller, Marius Molund, Tor Nicolaisen, Fredrik Nilsen, Jonas Rydinge, Morten Smedsrud, Are Stødle, Axel Trommer, Stein Ugland, Anders Karlsten, Guri Ekås, Elise Berg Vesterhus, Anne Christine Brekke (Oslo University Hospital, Norway); Ajay Gupta, Neeraj Jain, Farah Khan (Nirmal Hospital, India); Ateet Sharma, Amir Sanghavi, Mittal Trivedi (Satellite Orthopaedic Hospital and Research Centre, India); Anil Rai, Subash, Kamal Rai (Highway Hospital, India); Vineet Yadav, Sanjay Singh, Kamal Rai (Popular Hospital, India); Kevin Tetsworth, Geoff Donald, Patrick Weinrauch, Paul Pincus, Steven Yang, Brett Halliday, Trevor Gervais, Michael Holt, Annette Flynn (Royal Brisbane and Women's Hospital, Australia); Amal Shankar Prasad, Vimlesh Mishra (Madhuraj Nursing Home, India); D. C. Sundaresh, Angshuman Khanna (M. S. Rammaiah Medical College \& Hospital, India); Joe Joseph Cherian, Davy J Olakkengil, Gaurav Sharma (St John's Medical College Hospital, India); Marinis Pirpiris, David Love, Andrew Bucknill, Richard J Farrugia (Royal Melbourne Hospital, Australia); HansChristoph Pape, Matthias Knobe, Roman Pfeifer (University of Aachen Medical Center, Germany); Peter Hull, Sophie Lewis, Simone Evans (Cambridge University Hospitals, England); Rajesh Nanda, Rajanikanth Logishetty, Sanjeev Anand, Carol Bowler (University Hospital of North Tees, England); Akhil Dadi, Naveen Palla, Utsav Ganguly (Sunshine Hospital, India); B. Sachidananda Rai, Janakiraman Rajakumar (Unity Health Complex, India); Andrew Jennings, Graham Chuter, Glynis Rose, Gillian Horner (University Hospital of North Durham and Darlington Memorial Hospital, England); Callum Clark, Kate Eke (Wexham Park Hospital, England); Mike Reed, Chris Herriott, Christine Dobb (Northumbria Healthcare NHS Foundation Trust, England).

\section{References}

1. Bhandari M, Chiavaras M, Ayeni O, Chakraverrty R, Parasu N, Choudur H, Bains S, Sprague S, Petrisor B. Assessment of radiographic fracture healing in patients with operatively treated femoral neck fractures. J Orthop Trauma. 2013;27:e213-219.

2. Bhandari M, Chiavaras MM, Parasu N, Choudur H, Ayeni O, Chakravertty R, Bains S, Hak A, Sprague S, Petrisor B.
Radiographic union score for hip substantially improves agreement between surgeons and radiologists. BMC Musculoskelet Disord. 2013;14:70.

3. Blomfeldt R, Tornkvist H, Ponzer S, Soderqvist A, Tidermark J. Comparison of internal fixation with total hip replacement for displaced femoral neck fractures. Randomized, controlled trial performed at four years. J Bone Joint Surg Am. 2005;87:16801688.

4. Brinker MR. Nonunions: evaluation and treatment. In: Browner BD, Levine AM, Trafton PG, eds. Skeletal Trauma: Basic Science, Management, and Reconstruction. Philadelphia, PA, USA: WB Saunders; 2003:98-199.

5. Chiavaras MM, Bains S, Choudur H, Parasu N, Jacobson J, Ayeni O, Petrisor B, Chakravertty R, Sprague S, Bhandari M. The Radiographic Union Score for Hip (RUSH): the use of a checklist to evaluate hip fracture healing improves agreement between radiologists and orthopedic surgeons. Skeletal Radiol. 2013;42:1079-1088.

6. Corrales LA, Morshed S, Bhandari M, Miclau T $3^{\text {rd }}$. Variability in the assessment of fracture-healing in orthopaedic trauma studies. J Bone Joint Surg Am. 2008;90:1862-1868.

7. Elmerson S, Sjostedt A, Zetterberg C. Fixation of femoral neck fracture. A randomized 2-year follow-up study of hook pins and sliding screw plate in 222 patients. Acta Orthop Scand. 1995;66:507-510.

8. FAITH Investigators. Fixation using alternative implants for the treatment of hip fractures (FAITH): design and rationale for a multi-centre randomized trial comparing sliding hip screws and cancellous screws on revision surgery rates and quality of life in the treatment of femoral neck fractures. BMC Musculoskelet Disord. 2014;15:219.

9. Gullberg B, Johnell O, Kanis JA. World-wide projections for hip fracture. Osteoporos Int. 1997;7:407-413.

10. Health Care Financing Administration. Coverage issues. In: Department of Health and Human Services, eds. Medical Procedures Manual. Baltimore, MD, USA: Centers for Medicare and Medicaid Services; 2000:35-51.

11. Johansson T, Jacobsson SA, Ivarsson I, Knutsson A, Wahlstrom O. Internal fixation versus total hip arthroplasty in the treatment of displaced femoral neck fractures: a prospective randomized study of 100 hips. Acta Orthop Scand. 2000; 71:597-602.

12. Johnell O, Kanis JA. An estimate of the worldwide prevalence, mortality and disability associated with hip fracture. Osteoporos Int. 2004;15:897-902.

13. Landis JR, Koch GG. The measurement of observer agreement for categorical data. Biometrics. 1977;33:159-174.

14. Liporace FA, Egol KA, Tejwani N, Zuckerman JD, Koval KJ. What's new in hip fractures? Current concepts. Am J Orthop (Belle Mead NJ). 2005;34:66-74.

15. Madsen F, Linde F, Andersen E, Birke H, Hvass I, Poulsen TD. Fixation of displaced femoral neck fractures. A comparison between sliding screw plate and four cancellous bone screws. Acta Orthop Scand. 1987;58:212-216.

16. Wihlborg O. Fixation of femoral neck fractures. A four-flanged nail versus threaded pins in 200 cases. Acta Orthop Scand. 1990;61:415-418. 